\title{
Introduction to special section on digital technology and cancer survivorship
}

\author{
Kate Absolom $^{1}$ (D) Patricia Holch ${ }^{1,2} \cdot$ Ziv Amir $^{3}$
}

Received: 24 August 2017 / Accepted: 29 August 2017 /Published online: 14 September 2017

(C) Springer Science+Business Media, LLC 2017

\section{Introduction}

Technological advances in health care have led to increasing numbers of curable and treatable cancers extending the lives of many people around the world. Alongside these advances, the development and accessibility of modern technology including the internet, social media, smart phones and apps are revolutionising how we communicate and receive information and store and monitor data. This in turn has led to novel methods of supporting people living with and beyond cancer. In this special section of the Journal of Cancer Survivorship, we are pleased to present a collection of seven papers showcasing a variety of technologies and research methodologies. Each demonstrates the utilisation of different digital approaches for either improving our understanding of the lives of people surviving cancer or assisting the management of the consequences of the disease. Collectively, the papers raise a number of important questions for future exploration as we adapt to an ever more digitally focussed society.

Kate Absolom

k.1.absolom@leeds.ac.uk

1 Section of Patient Centred Outcomes Research, Leeds Institute of Cancer and Pathology, University of Leeds, Leeds, UK

2 Psychology Group, School of Social Sciences, Faculty of Health and Social Sciences, Leeds Beckett University, Leeds, UK

3 Cancer Rehabilitation, Wellness Works, University of Salford, Salford, UK

\section{Information and peer support}

Two of the papers selected for this special section describe cancer survivors' views and use of the internet and online resources to meet medical information needs and foster interactions with contemporaries. In their in depth qualitative exploration of how adult cancer survivors discuss navigating the internet, Harkins et al. [1] describe a number of perceived positive benefits including the potential facilitation of reframing survivors' post diagnosis identity. Abrol and colleagues [2] present findings from a survey of technology preferences among young cancer survivors (aged 13-24 years). As the authors acknowledge, this generation is heralded as "digital natives": therefore, the perceived importance of online communication was high in this group. Both these papers highlight the ongoing need for health care systems and policy makers to work towards clear recommendations on the use of webbased resources to help meet survivors' informational needs and improve clinical interactions. In addition, it is becoming increasingly important that survivors are routinely directed to reputable internet sites and guidance is provided to help equip individuals with the skills needed to assess the standard and relevance of websites and online forums they may access.

\section{Challenges of integrating electronic patient assessment}

McNeill and colleagues' study [3] also focuses on young cancer survivors. In this feasibility work, the views of survivors of retinoblastoma, parents and clinicians on the incorporation of an electronic Patient Reported Outcomes Measures (PROMs) system into routine follow-up were assessed. The integration of PROMs into clinical practice is an area that has received considerable attention in recent years as the value and importance 
of providing patient centred care has been increasingly recognised. Here McNeill et al.'s paper describes some of the potential benefits and ongoing challenges with establishing electronic PROM assessments, from decisions around the most relevant and appropriate assessment measure to use to the technical complexities of collecting patient reports and displaying data to clinicians within hospital computer systems.

\section{Improving survivor outcomes}

The remaining four papers chosen for this special section address the development or evaluation of digital interventions for improving survivors' health and wellbeing. Willems et al.'s [4] paper reports secondary analysis from a randomised trial exploring the longer term outcomes of a web-based psychosocial support intervention for cancer survivors. The results show the complexity of determining how and when different types of patient and demographic groups may benefit from specific interventions. In their systematic review and meta-analysis, Roberts et al. [5] reveal some encouraging evidence supporting the benefits of digital behaviour change interventions on survivors' physical activity and weight but ultimately conclusions remain limited by the heterogeneity of studies and the paucity of quality RCTs to date. It is expected that the burgeoning interest in mobile technology will lead to more quality research in this field in the near future. Roberts et al. [5] also conclude that improved understanding of strategies for enhancing patient engagement with digital interventions to help maintain adherence is necessary. This is a salient issue within the exercise intervention feasibility studies provided by Timmerman et al. [6] (lung cancer survivors) and Harder et al. [7] (breast cancer survivors) where the value of putting patient user input at the centre of the design and assessment of digital interventions is clearly demonstrated. We are pleased to include these studies as they also reveal the extent of developmental work required to design and implement electronically supported interventions.

\section{Conclusions}

Collectively, these research papers emphasise the many ways in which digital technology is being assimilated into the lives of cancer survivors. At a broad level, technology has been heralded as an ideal platform to provide cost-effective methods for delivering medical information, social support and interventions to large numbers of cancer survivors and other patient groups. This special section highlights the benefits of technology and current limitations in our understanding of how it can best be used to optimise outcomes for cancer survivors.

As internet access becomes increasingly commonplace it would be beneficial to see the availability of guidelines and recommendations to assist with directing survivors towards quality controlled medical and social websites and the promotion of digital literacy. Methods for capturing and utilising electronic patient reported outcomes measures (PROMs) are also rising as health systems become more technologically focussed. PROMs data have the potential at both an individual and population level to benefit survivors and broaden understanding of the impact of cancer. The development and wider use of adaptive reporting systems throughout the cancer trajectory that are systematically integrated into electronic patient records and portals would be advantageous.

Digital health behaviour and psychosocial interventions come in a variety of configurations and guises but further large scale robust evaluation studies are needed to accurately determine how effective these tools are for meeting the needs of different survivor groups. Certainly the importance of placing user input from patients and health professionals at the forefront of intervention development and assessment is evident. Further exploration of the psychological processes and theoretical underpinnings of how digital technologies may work to effectively empower survivors and improve outcomes are also warranted.

It is certainly an exciting time for technology and cancer survivorship as health care services continue evolving to incorporate new electronic developments. Coming years will undoubtedly see a major rise in published studies in this area that will add to the current knowledge base and assist with providing further technological benefit to those affected by a cancer diagnosis.

\section{Compliance with ethical standards}

Conflict of interest The authors declare that they have no conflicts of interest.

\section{References}

1. Harkin LJ, Beaver K, Dey P, Choong K. Navigating cancer using online communities: a grounded theory of survivor and family experiences. $\mathrm{J}$ Cancer Surviv. 2017. https://doi.org/10.1007/s11764-017-0616-1.

2. Abrol E, Groszmann M, Pitman A, Hough R, Taylor RM, Aref-Adib G. Exploring the digital technology preferences of teenagers and young adults (TYA) with cancer and survivors: a cross-sectional service evaluation questionnaire. J Cancer Surviv. 2017. https://doi. org/10.1007/s11764-017-0618-z.

3. McNeill NA, Kors WA, Bosscha MI, van Dijk J, Fabius AWM, Ton Houffelaar T, Verdonck-de Leeuw IM, Moll MC. Feasibility of RetinoQuest: e-health application to facilitate and improve additional care for retinoblastoma survivors. J Cancer Surviv. 2017. https://doi. org/10.1007/s11764-017-0642-z.

4. Willems RA, Mesters I, Lechner L, Kanera IM, Bolman CAW. Longterm effectiveness and moderators of a web-based tailored intervention for cancer survivors on social and emotional functioning, depression, and fatigue: randomized controlled trial. J Cancer Surviv. 2017. https://doi.org/10.1007/s11764-017-0625-0.

5. Roberts AL, Fisher A, Smith L, Heinrich M, Potts HWW. Digital health behaviour change interventions targeting physical activity and 
diet in cancer survivors: a systematic review and meta-analysis. $\mathrm{J}$ Cancer Surviv. 2017. https://doi.org/10.1007/s11764-017-0632-1.

6. Timmerman JG, Dekker-van Weering MG, Stuiver MM, Groen WG,

Wouters MW, Tonis TM, et al. Ambulant monitoring and webaccessible home-based exercise program during outpatient followup for resected lung cancer survivors: actual use and feasibility in clinical practice. J Cancer Surviv. 2017. https://doi.org/10.1007/ s11764-017-0611-6.

7. Harder H, Holroyd P, Burkinshaw L, Watten P, Zammit C, Harris PR, et al. A user-centred approach to developing bWell, a mobile app for arm and shoulder exercises after breast cancer treatment. J Cancer Surviv. 2017. https://doi.org/10.1007/s11764-017-0630-3. 